\title{
Optimal coupling of heat and electricity systems: A stochastic hierarchical approach
}

\author{
Mitridati, Lesia Marie-Jeanne Mariane; Pinson, Pierre
}

Published in:

Proceedings of International Conference on Probabilistic Methods Applied to Power Systems

Link to article, DOI:

10.1109/PMAPS.2016.7764188

Publication date:

2016

Document Version

Peer reviewed version

Link back to DTU Orbit

Citation $(A P A)$ :

Mitridati, L. M-J. M., \& Pinson, P. (2016). Optimal coupling of heat and electricity systems: A stochastic hierarchical approach. In Proceedings of International Conference on Probabilistic Methods Applied to Power Systems IEEE. https://doi.org/10.1109/PMAPS.2016.7764188

\section{General rights}

Copyright and moral rights for the publications made accessible in the public portal are retained by the authors and/or other copyright owners and it is a condition of accessing publications that users recognise and abide by the legal requirements associated with these rights.

- Users may download and print one copy of any publication from the public portal for the purpose of private study or research.

- You may not further distribute the material or use it for any profit-making activity or commercial gain

- You may freely distribute the URL identifying the publication in the public portal 


\title{
Optimal Coupling of Heat and Electricity Systems: A Stochastic Hierarchical Approach
}

\author{
Lesia Mitridati and Pierre Pinson \\ Dept. of Electrical Engineering \\ Technical University of Denmark \\ Kgs. Lyngby 2800 \\ Email: lemitri@elektro.dtu.dk ppin@elektro.dtu.dk
}

\begin{abstract}
The large penetration of renewables in the power system increases the need for flexibility. Flexibility gains and wind curtailment reduction can be achieved through a better coordination with other energy systems, in particular with district heating. Loose interactions between these two systems already exist due to the participation of CHPs in both markets. New market structures must be developed in order to exploit these synergies. Recognizing the above-mentioned challenges this paper proposes a stochastic hierarchical formulation of the heat economic dispatch problem in a system with high penetration of CHPs and wind. The objective of this optimization problem is to minimize the heat production cost, subject to constraints describing day-ahead electricity market clearing scenarios. Uncertainties concerning wind power production, electricity demand and rival participants offers are efficiently modelled using a finite set of scenarios. This model takes advantage of existing market structures and provides a decision-making tool for heat system operators. The proposed model is implemented in a case study and results are discussed to show the benefits and applicability of this approach.
\end{abstract}

Keywords-Integrated energy systems, district heating, hierarchical optimization, MPEC, stochastic programming

\section{NOMENCLATURE}

\section{Indexes}

$t \quad$ Index for hours

$g \quad$ Index for electricity generation units

$w \quad$ Index for wind turbines

$c \quad$ Index for CHP units

$c \in \mathcal{X}^{E} \quad$ Set of extraction CHP units

$c \in \mathcal{X}^{B} \quad$ Set of back-pressure CHP units

$h \quad$ Index for heat-only units

$\nu \quad$ Index for day-ahead electricity market scenarios

\section{Parameters}

$\pi_{\nu} \quad$ Probability of scenario $\nu[-]$

$L_{t}^{H} \quad$ Heat load [MWh]

$L_{t \nu}^{E} \quad$ Electricity load [MWh]

$\alpha_{c t}^{0} \quad$ Marginal cost of CHP unit $c[\$ / \mathrm{MWh}]$

$\alpha_{h t}^{0} \quad$ Marginal cost of heat-only unit $h$ [\$/MWh]

$\alpha_{c t}^{+}, \alpha_{c t}^{-} \quad$ Redispatch cost of CHP unit $c$ [\$/MWh]

$\alpha_{h t^{+}}, \alpha_{h t}^{-}$Redispatch cost of heat-only unit $h$ [\$/MWh]

$\alpha_{g t \nu} \quad$ Marginal cost of power plant $g$ [\$/MWh]

$\alpha_{w t \nu} \quad$ Marginal cost of wind farm $w$ [\$/MWh]

$\bar{F}_{c}$

$\bar{Q}_{h}$

$\bar{Q}_{c}$

$R_{c}^{D}$
$R_{h}^{D}$
$R_{c}^{U}$
$R_{h}^{U}$
$r_{c}$
$\rho_{c}^{H}$
$\rho_{c}^{E}$
$\bar{P}\left(Q_{c t}^{0}\right)$
$\bar{P}_{g t}$
$\bar{P}_{w t \nu}$

Ramp-down limit of CHP unit $c$ [MWh/h]

Ramp-down limit of heat-only unit $h[\mathrm{MWh} / \mathrm{h}]$

Ramp-up limit of CHP unit $c$ [MWh/h]

Ramp-up limit of heat-only unit $h[\mathrm{MWh} / \mathrm{h}]$

Electricity/heat ratio of CHP unit $c[-]$

Fuel/heat ratio of CHP unit $c[-]$

Fuel/electricity ratio of CHP unit $c[-]$

Maximum power output of CHP unit $c$ [MWh]

Maximum output of power plant $g$ [MWh]

Maximum power output of wind farm $w$ [MWh]

Upper-Level Variables

$Q_{c t}^{0} \quad$ Initial heat dispatch for CHP unit $c$ [MWh]

$Q_{h t}^{0} \quad$ Initial heat dispatch for heat-only unit $h$ [MWh]

$Q_{c t \nu}^{+}, Q_{c t \nu}^{-}$Heat redispatch for CHP unit $c$ [MWh]

$Q_{h t \nu}^{+}, Q_{h t \nu}^{-}$Heat redispatch for heat-only unit $h[\mathrm{MWh}]$

Lower-Level Variables

$P_{c t \nu} \quad$ Power dispatch for CHP unit $c$ [MWh]

$P_{g t \nu} \quad$ Power dispatch for power plant $g$ [MWh]

$P_{w t \nu} \quad$ Power dispatch for wind farm $w$ [MWh]

$\lambda_{t \nu}^{s p o t} \quad$ Spot price $[\$ / \mathrm{MWh}]$

\section{INTRODUCTION}

Due to growing environmental concerns and economic incentives renewable energy sources such as wind power have known a rapid growth. This large share of stochastic and nondispatchable energy sources increases the need for flexibility in the power system. This issue can be addressed by achieving closer interaction with other energy systems, such as district heating [1], [2].

Combined heat and power units (CHPs) participate in both heat and electricity markets. In systems with high penetration of CHPs, the linkage between heat and power outputs imposes strong constraints to the electricity system. As a matter of fact CHPs' dispatch is traditionally driven by heat demand, while electricity is managed as a byproduct. This limited flexibility of CHPs can be a barrier for a large-scale penetration of stochastic generation as wind and solar power [3], [4].

These studies show that it is fundamental to develop new market structures in order to achieve an optimal coupling of heat and electricity systems and increase the flexibility of the overall system. [5] proposes a joint heat and power dispatch exploiting the existing storage capacity of district heating networks to increase the flexibility of CHPs. While [6] studies 
the optimal joint operation of heat storage and CHPs with a Nordic electricity market structure.

Moreover the large share of renewable energy sources increases the uncertainty and volatility in the electricity market. In order to deal with this issue, [7], [8] study the optimal dispatch of CHPs under uncertainty, using stochastic programming and robust optimization. These papers introduce uncertainty in the electricity market using electricity prices as exogenous uncertain parameters. However this approach doesn't allow us to model explicitly the influence of the participation of CHPs on both markets.

We propose a hierarchical optimization approach, where the heat dispatch is the upper-level optimization problem. The competition in the day-ahead electricity market is explicitly modelled in the lower-level problems. Hierarchical optimization, also referred to as mathematical problem with equilibrium constraints (MPEC), has found various applications in energy systems [9], [10]. Additionally we characterize the uncertainty in the day-ahead electricity market using uncertain wind production, electricity demand and rival participants bids.

This paper is organized as follows. Section II describes the proposed approach. Section III details the mathematical formulation consisting in a stochastic hierarchical model. Section IV provides the results of a case study. Finally, Section $\mathrm{V}$ concludes the paper with some relevant remarks.

\section{PRoblem DESCRIPTION}

\section{A. Heat and Electricity Systems Framework}

In this work we consider the problem of independent heat and electricity systems with high penetration of CHPs and wind production. In this framework, similar to the Copenhagen area, the day-ahead heat dispatch is performed by an independent heat system operator. Although the heat price is fixed based on long term contracts, the heat system operator prepares the heat dispatch in order to minimize heat production costs.

Due to the linkage between heat and electricity outputs, the cost allocation for heat and electricity production of CHPs is not straightforward. In order to solve this issue, the heat system operator defines the cost of heat as the total production cost minus the expected revenues from sales in the day-ahead electricity market

$$
\operatorname{Cost}\left(P_{c t}, Q_{c t}\right)-\lambda_{t}^{s p o t} P_{c t}
$$

However in the current framework, sales in the day-ahead electricity market are uncertain because the heat dispatch is performed before the day-ahead electricity market. A traditional approach is to use an expected value of the uncertain parameters $\lambda_{t}^{\text {spot }}$ and $P_{c t}$ to cost of heat.

After the heat requirements have been fixed, CHPs can participate to the day-ahead electricity market. Yet their available power output in the day-ahead electricity market is constrained by the scheduled heat production [11]. We can distinguish two main types of CHPs. Back-pressure units produce heat and electricity at a fixed ratio $r_{c}$ and their joint feasible operating region (FOR) for heat and electricity can be modelled as

$$
\begin{array}{ll}
P_{c t}=r_{c} Q_{c t} & \forall c \in \mathcal{X}^{B}, t \\
0 \leq Q_{c t} \leq \bar{Q}_{c} & \forall c \in \mathcal{X}^{B}, t .
\end{array}
$$

And extraction units allow more flexibility for heat and electricity production. The FOR of an extraction unit can be nonconvex and complex to model but we approximate it using a convex set of inequalities

$$
\begin{array}{ll}
0 \leq P_{c t} \geq r_{c} Q_{c t} & \forall c \in \mathcal{X}^{E}, t \\
0 \leq \rho_{c}^{H} Q_{c t}+\rho_{c}^{E} P_{c t} \leq \bar{F}_{c} & \forall c \in \mathcal{X}^{E}, t \\
0 \leq Q_{c t} \leq \bar{Q}_{c} & \forall c \in \mathcal{X}^{E}, t
\end{array}
$$

where Equation (3a) represents the minimum heat/power ratio, (3b) the maximum fuel intake and (3c) the maximum heat output. Figure 1 shows the simplified FOR of an extraction unit.

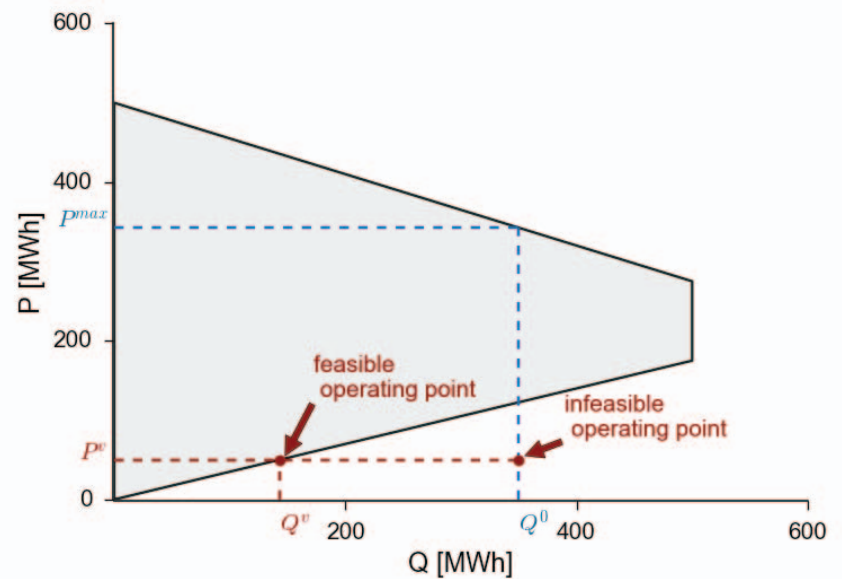

Fig. 1. Simplified FOR of an extraction unit

\section{B. Hierarchical Setup}

Our aim is to develop a decision-making tool for an independent heat system operator. Its primary objective is to minimize the cost of heat, as defined in (1). Co-clearing approaches proposed in the literature [5], aiming at minimizing the overall system production cost, are not compatible with this imposed framework. The merit order in the heat market depends on the revenues in the day-ahead electricity market, which are uncertain. Equilibrium models [12] allow us to model the interaction between different players, yet they do not account for the sequential decision-making of the heat and electricity market operators. We propose a method that follows the existing sequential heat and electricity market clearing order. In addition, we consider a system with high penetration of CHP plants. Hence the impact of the heat dispatch on the electricity market should not be neglected. That is why it is 
essential to model explicitly the participation of CHPs in the electricity market. Whereas single-level optimization models, introducing spot prices as exogenous uncertain variables, do not allow us to model accurately this dependency.

We formulate the day-ahead heat dispatch as a hierarchical optimization problem, in which the heat operator is the leader. In the upper-level problem, the heat operator seeks to minimize the cost of heat, while anticipating on the reaction of the followers in the day-ahead electricity market. The day-ahead electricity market clearing is explicitly modelled in the lowerlevel optimization problems. The hierarchical setup of this problem is outlined in Figure 2.

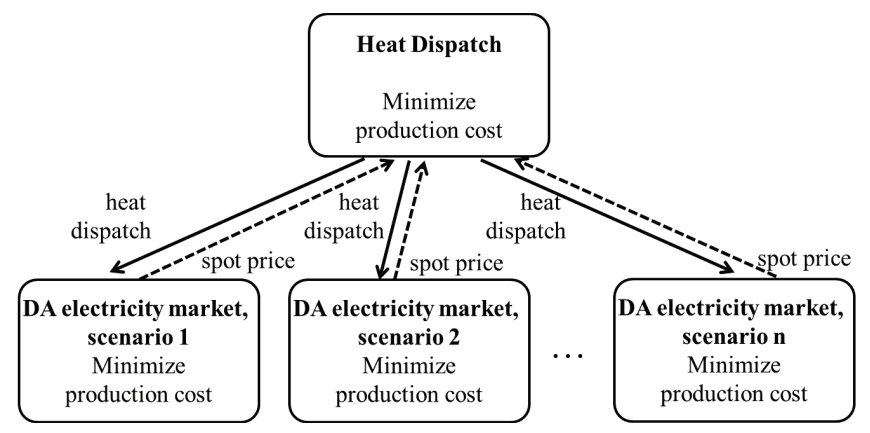

Fig. 2. Sketch of the hierarchical optimization setup

Additionally the uncertainty on the electricity market clearing is introduced through three exogenous parameters, wind production, electricity demand and other participants bids. We model this uncertainty using a finite set of scenarios and use stochastic programming to solve the heat dispatch problem. For simplicity we denote $\nu$ the set of scenarios for all parameters and $\pi_{\nu}$ their probability.

\section{MATHEMATICAL FORMULATION}

\section{A. Standard Stochastic Hierarchical Formulation}

The standard form of the two-stage stochastic MPEC introduced above can be expressed as

$$
\begin{array}{lll}
\min _{\Omega} & f^{0}\left(Q^{0}\right)+\mathbb{E}\left(f\left(Q_{\nu}^{+}, Q_{\nu}^{-}, P_{\nu}, \lambda_{\nu}, \mu_{\nu}\right)\right) \\
\text { s.t. } & h^{0}\left(Q^{0}\right) \leq 0 & \\
& h\left(Q^{0}, Q_{\nu}^{+}, Q_{\nu}^{-}, P_{\nu}\right) \leq 0 & \forall \nu \\
& \left(P_{\nu}, \lambda_{\nu}, \mu_{\nu}\right) \in \underset{P_{\nu} \in H\left(Q^{0}\right)}{\arg \min }\left\{F\left(P_{\nu}\right)\right\} & \forall \nu
\end{array}
$$

where $\Omega=\left\{Q^{0}, Q_{\nu}^{+}, Q_{\nu}^{-}\right\} \bigcup\left\{P_{\nu}, \lambda_{\nu}, \mu_{\nu}\right\}$ is the set of upperlevel and lower-level variables. And for simplicity, in this section we omit the indexes representing the production units and time steps.

The upper-level problem consists in the minimization of the objective function (4a), subject to constraints (4b) and (4c) and the optimality of the lower level problems (4d). The decision variables of the upper-level problem $Q^{0}$ represent the initial heat dispatch. Additionally we consider that CHPs are allowed to default on the initial heat dispatch if it is not compatible with the electricity production dispatched in the day-ahead electricity market, as shown in Figure 1. The recourse variables $Q_{\nu}^{+}$and $Q_{\nu}^{-}$represent the redispatch of CHPs and heat-only units. The upper-level objective function also depends on the primal and dual variables $P_{\nu}, \lambda_{\nu}, \mu_{\nu}$ of the lower-level problems (4d). This is due to the dependency of the heat cost on the revenues from electricity sales in the day-ahead electricity market as expressed in (1). Additionally the upper-level constraints $(4 \mathrm{c})$ link the upper-level variables $Q^{0}, Q_{\nu}^{+}, Q_{\nu}^{-}$to the lower-level variables $P_{\nu}$, due to the linkage between heat and electricity outputs of CHPs (2)-(3).

The lower-level optimization problems $(4 \mathrm{~d})$ represent the day-ahead electricity market clearing for each scenario $\nu . P_{\nu}$ represents the primal variables of the lower-level problems and $\lambda_{\nu}, \mu_{\nu}$ represent their dual variables. The structure of the upper and lower-level problems are further detailed in section III-B.

\section{B. Lower-Level Problems}

For a given scenario $\nu$, the lower-level problem expressed in (4d) solves the following simplified day-ahead electricity market clearing

$$
\begin{array}{cc}
\min _{\Gamma} \sum_{g t} \alpha_{g t \nu} P_{g t \nu}+\sum_{w t} \alpha_{w t \nu} P_{w t \nu}+\sum_{c t} \alpha_{c t}^{0} \rho_{c}^{E} P_{c t \nu} \\
\text { s.t. } \quad L_{t \nu}^{E}=\sum_{g} P_{g t \nu}+\sum_{c} P_{c t \nu} & \\
+\sum_{w} P_{w t \nu} & \forall t \quad: \lambda_{t \nu}^{s p o t} \\
0 \leq P_{g t \nu} \leq \bar{P}_{g t} & \forall g, t: \underline{\mu}_{g t \nu}, \bar{\mu}_{g t \nu} \\
\left.0 \leq P_{c t \nu} \leq \bar{P}_{\left(Q_{c t}\right.}\right) & \forall c, t: \underline{\mu}_{c t \nu}, \bar{\mu}_{c t \nu} \\
0 \leq P_{w t \nu} \leq \bar{P}_{w t \nu} & \forall w, t: \underline{\mu}_{w t \nu}, \bar{\mu}_{w t \nu}
\end{array}
$$

where $\Gamma=\left\{P_{c t \nu}, P_{g t \nu}, P_{w t \nu}\right\}$ is the set of primal lower-level variables and $\Xi=\left\{\lambda_{t \nu}^{s p o t}, \underline{\mu}_{g t \nu}, \bar{\mu}_{g t \nu}, \underline{\mu}_{c t \nu}, \bar{\mu}_{c t \nu}, \underline{\mu}_{w t \nu}, \bar{\mu}_{w t \nu}\right\}$ the set of dual lower-level variables. In addition we define the maximum power available for CHP $c$ to bid in the day-ahead electricity market as a function of the initial heat dispatch

$$
\bar{P}\left(Q_{c t}^{0}\right)= \begin{cases}\frac{\bar{F}_{c}-\rho_{c}^{H} Q_{c t}^{0}}{\rho_{c t}^{E}} & \forall c \in \mathcal{X}^{E}, t, \\ r_{c} Q_{c t}^{0} & \forall c \in \mathcal{X}^{B}, t .\end{cases}
$$

In the lower-level problems $Q_{c t}^{0}$ and $\bar{P}\left(Q_{c t}^{0}\right)$ are considered fixed. The lower-level optimization problem for scenario $\nu$ consists in minimizing the electricity production cost $(5 \mathrm{a})$, while enforcing power balance (5b) and constrained by the generators maximum power output of thermal power generators, CHPs and wind turbines (5c)-(5e). Since this problem is linear in the continuous variables $P_{g t \nu}, P_{w t \nu}$, and $P_{c t \nu}$, its Karush-Kuhn-Tucker (KKT) conditions are necessary and sufficient. And it can be reformulated as 


$$
\begin{array}{lll}
\sum_{g} P_{g t \nu}+\sum_{c} P_{c t \nu}+\sum_{w} P_{w t \nu}=L_{t \nu}^{E} & \forall t \\
\alpha_{g t \nu}-\lambda_{t \nu}^{s p o t}+\bar{\mu}_{g t \nu}-\underline{\mu}_{g t \nu} & =0 & \forall g, t \\
\alpha_{c t} \rho_{c}^{E}-\lambda_{t \nu}^{s p o t}+\bar{\mu}_{c t \nu}-\underline{\mu}_{c t \nu} & =0 & \forall c, t \\
\alpha_{w t \nu}-\lambda_{t \nu}^{s p o t}+\bar{\mu}_{w t}-\underline{\mu}_{w t} & =0 & \forall w, t \\
0 \leq \bar{\mu}_{g t \nu} \perp\left(P_{g t \nu}-\bar{P}_{g t}\right) & \leq 0 & \forall g, t \\
0 \leq \underline{\mu}_{g t \nu} \perp\left(-P_{g t \nu}\right) & \leq 0 & \forall g, t \\
0 \leq \bar{\mu}_{c t \nu} \perp\left(P_{c t \nu}-\bar{P}\left(Q_{c t}^{0}\right)\right) & \leq 0 & \forall c, t \\
0 \leq \underline{\mu}_{c t \nu} \perp\left(-P_{c t \nu}\right) & \leq 0 & \forall c, t \\
0 \leq \bar{\mu}_{w t \nu} \perp\left(P_{w t \nu}-\bar{P}_{w t \nu}\right) & \leq 0 & \forall w, t \\
0 \leq \underline{\mu}_{w t \nu} \perp\left(-P_{w t \nu}\right) & \leq 0 & \forall w, t
\end{array}
$$

where (6b)-(6d) represent the stationarity conditions, and (6e)-(6j) the complementarity conditions corresponding to the inequality constraints (5c)-(5e). Additionally the complementarity conditions can be linearised using binary variables [13] or relaxed using an SOS1-based approach [14].

\section{Upper-Level Problem}

The upper-level optimization problem represents the optimal day-heat dispatch problem. We consider a system with extraction and back-pressure CHPs and heat-only units. We neglect transmission constraints as well as start-up and shutdown costs. This problem can be formulated as follows

$$
\begin{aligned}
& \min _{\Theta \cup\{\Gamma, \Xi\}} \sum_{h t} \alpha_{h t}^{0} Q_{h t}^{0}+\sum_{c t} \alpha_{c t}^{0} \rho_{c}^{H} Q_{c t}^{0} \\
& +\sum_{\nu} \pi_{\nu}\left[\sum_{c t}\left(\alpha_{c t}^{0} \rho_{c}^{E} P_{c t \nu}-\lambda_{t \nu}^{s p o t} P_{c t \nu}\right)\right. \\
& +\sum_{h t}\left(\alpha_{h t}^{+} Q_{h t \nu}^{+}-\alpha_{h t}^{-} Q_{h t \nu}^{-}\right) \\
& \left.+\sum_{c t}\left(\alpha_{c t}^{+} Q_{c t \nu}^{+}-\alpha_{c t}^{-} Q_{c t \nu}^{-}\right)\right]
\end{aligned}
$$

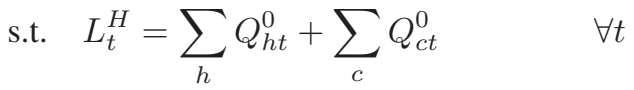

$$
\begin{aligned}
& 0 \leq Q_{c t}^{0} \leq \bar{Q}_{c} \quad \forall c, t \\
& 0 \leq Q_{h t}^{0} \leq \bar{Q}_{h} \quad \forall h, t \\
& -R_{c}^{D} \leq Q_{c(t+1)}^{0}-Q_{c t}^{0} \leq R_{c}^{U} \quad \forall c, t \\
& -R_{h}^{D} \leq Q_{h(t+1)}^{0}-Q_{h t}^{0} \leq R_{h}^{U} \quad \forall h, t \\
& L_{t}^{H}=\sum_{h} Q_{h t \nu}+\sum_{c} Q_{c t \nu} \quad \forall t, \nu \\
& 0 \leq Q_{h, t, \nu} \leq \bar{Q}_{h} \quad \forall h, t, \nu \\
& -R_{c}^{D} \leq Q_{c(t+1) \nu}-Q_{c t \nu} \leq R_{c}^{U} \quad \forall c, t, \nu \\
& -R_{h}^{D} \leq Q_{h(t+1) \nu}-Q_{h t \nu} \leq R_{h}^{U} \quad \forall h, t, \nu \\
& Q_{c, t, \nu} \in \mathcal{D}\left(P_{c t \nu}\right) \quad \forall c, t, \nu \\
& 0 \leq Q_{c t \nu}^{+}, Q_{c t \nu}^{-}, Q_{h t \nu}^{+}, Q_{h t \nu}^{-} \quad \forall h, c, t, \nu \\
& (6 \mathrm{a})-(6 \mathrm{j}) \quad \forall \nu
\end{aligned}
$$

where $\Theta=\left\{Q_{c t}^{0}, Q_{h t}^{0}, Q_{c t \nu}^{+}, Q_{c t \nu}^{-}, Q_{h t \nu}^{+}, Q_{h t \nu}^{-}\right\}$is the set of upper-level variables. $\Gamma$ and $\Xi$ are the sets of lower-level variables. For simplicity we defined two auxiliary variables representing the final heat dispatch of CHPs and heat-only units

$$
\begin{aligned}
Q_{c t \nu} & =Q_{c t}^{0}+Q_{c t \nu}^{+}-Q_{c t \nu}^{-} \quad \forall c, t, \nu, \\
Q_{h t \nu} & =Q_{h t}^{0}+Q_{h t \nu}^{+}-Q_{h t \nu}^{-} \quad \forall h, t, \nu .
\end{aligned}
$$

And $\mathcal{D}\left(P_{c t \nu}\right)$ represents the FOR of CHP $c$, as defined in (2) and (3), expressed as a function of the electricity output $P_{c t \nu}$.

The objective of this optimization problem is to minimize the expected cost of heat (7a), calculated as initial heat dispatch and redispatch costs, minus expected revenues from the day-ahead electricity market. We approximate the production cost of heat-only units and CHPs as linear functions, proportional to the total fuel consumption. The first stage constraints represent the initial heat balance (7b), heat production limits (7c)-(7d), and ramping constraints (7e)-(7f). Equations (7g)(7j) enforce the same constraints for the recourse variables in each scenario. In addition (7k) enforces that the final heat dispatch $Q_{c t \nu}$ is in the FOR of CHP $c$. This constraint links lower and upper-levels variables. Finally $(7 \mathrm{~m})$ represents the lowerlevel optimization problems, replaced by their equivalent KKT conditions.

Due to the bilinear terms $\lambda_{t}^{\text {spot }} P_{c t \nu}$ the upper-level problem is non-linear. We provide below an exact linearisation of the objective function based on the expression of the KKT conditions (6a)-(6j) and the strong duality theorem of the lower-level problems [12]:

$$
\begin{aligned}
\sum_{c t} \lambda_{t \nu}^{s p o t} P_{c t \nu}= & -\sum_{w t}\left(\alpha_{w t \nu} P_{w t \nu}+\bar{\mu}_{w t \nu} \bar{P}_{w t \nu}\right) \\
& +\sum_{t} \lambda_{t \nu}^{s p o t} L_{t \nu}^{E}-\sum_{g t}\left(\alpha_{g t \nu} P_{g t \nu}+\bar{\mu}_{g t \nu} \bar{P}_{g t}\right) .
\end{aligned}
$$

\section{CASe Study}

\section{A. Case Study Setup}

In this case study we consider a system comprising one wind producer $W_{1}$, two thermal power plants $\left(G_{1}, G_{2}\right)$, one back-pressure unit $C H P_{1}$, one extraction unit $C H P_{2}$ and one heat-only unit $H_{1}$. The back-pressure unit is cheaper than the extraction unit but it is less flexible due to its fixed electricity/heat ratio and ramping limits. The heat-only unit is more expensive than both CHPs and completely flexible. And the marginal cost of both thermal power plants are considered uncertain. The technical specifications of these units are listed in Table I. These parameters are loosely derived from values for existing units of the Copenhagen area as reported in [7].

A major challenge in stochastic programming is to model accurately the probability density of the uncertain parameters in order to generate appropriate scenarios. The uncertain parameters of this model, namely wind production, electricity demand and thermal power plants' bids, are assumed independent. For simplicity in this case study we assume that the 
TABLE I

HEAT AND ELECTRICITY SYSTEMS PARAMETERS

\begin{tabular}{ccccccc}
\hline & $C H P_{1}$ & $C^{2} P_{2}$ & $H_{1}$ & $W_{1}$ & $G_{1}$ & $G_{2}$ \\
\hline $\bar{F}$ & 500 & 500 & - & - & - & - \\
$\bar{P}$ & - & - & - & 400 & 500 & 500 \\
$\bar{Q}$ & 250 & 500 & 1000 & - & - & - \\
$R^{U}$ & 150 & 150 & 1000 & - & - & - \\
$R^{D}$ & 150 & 150 & 1000 & - & - & - \\
$Q^{\text {init }}$ & 0 & 400 & 0 & - & - & - \\
$\rho^{H}$ & 0.25 & 0.4 & - & - & - & - \\
$\rho^{E}$ & 2.4 & 2.4 & - & - & - & - \\
$\mathrm{r}$ & 0.6 & 0.25 & - & - & - & - \\
$\alpha^{0}$ & 25 & 12.5 & 100 & 0 & 45 & 65 \\
$\alpha^{+}$ & 50 & 25 & 125 & - & - & - \\
$\alpha^{-}$ & 10 & 5 & 100 & - & - & - \\
\hline
\end{tabular}

electricity demand is perfectly known, and we model electricity producers' marginal costs as mutually independent normal distributions. The parameters of these probability distributions are shown in Table I. We use a set of wind production scenarios provided at [15].

For heat demand we use a modified version of data from the western Copenhagen area provided in [16]. Figure 3 shows the heat load and electricity net loads for each scenario $\nu$ of wind production.

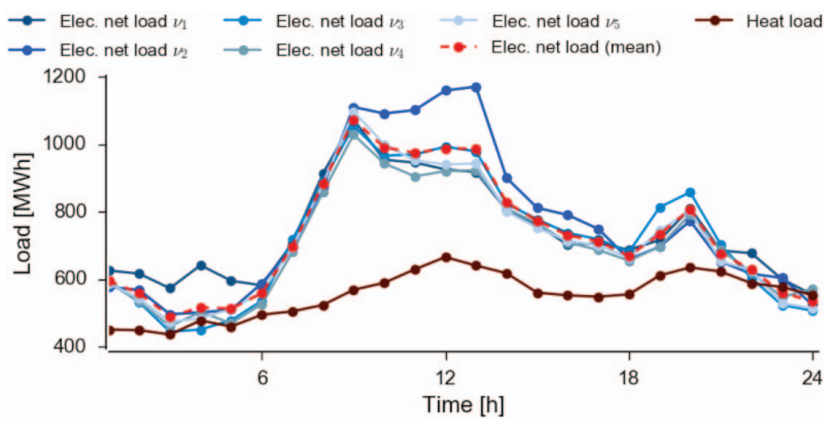

Fig. 3. Hourly heat load and electricity net load scenarios

\section{B. Simulation Results}

We implement the stochastic hierarchical model proposed in (7) on this system. And in order to assess the benefits of this method, we compare the results to a nominal hierarchical model $^{1}$. The nominal hierarchical model is the deterministic version of model (7), where all uncertain parameters are fixed at their expected value. We solve sequentially the initial heat dispatch, day-ahead electricity market, and heat redispatch for each realization of the day-ahead electricity market scenarios. Table II compares the average cost of heat over our set of scenarios for both models. It shows that the stochastic hierarchical model improves the average cost of heat by $3.8 \%$ compared to the nominal model. Note that this cost is a virtual cost used by the heat operator to evaluate the merit order of CHPs in the heat market (1). The actual heat production cost can be computed as the difference between total and

\footnotetext{
${ }^{1}$ The code for this optimization problem is available at [17]
}

electricity production costs in Table II. With the stochastic approach it is reduced by $2.6 \%$. However the reduction of the electricity production cost is negligible. This can be explained by the assumption that CHPs are not strategic in the day-ahead electricity market and block orders are not modelled.

TABLE II

RESUlTS: AVERAGE PRODUCTION COSTS $\left[10^{3} \$\right]$

\begin{tabular}{lcc} 
& Stochastic MPEC & Nominal MPEC \\
\hline Cost of heat & 56.41 & 58.68 \\
Electricity production cost & 763.93 & 764.18 \\
Total production cost & 901.71 & 905.60 \\
\hline
\end{tabular}

Figure 4 shows the initial heat dispatch for both models. In the stochastic model the extraction CHP is less dispatched than in the nominal one. This is due to the occurrence of certain scenarios where the extraction unit is not competitive in the day-ahead electricity market. The hierarchical structure allows us to anticipate on these scenarios and calculate accurately the merit order of the CHPs in the heat market.

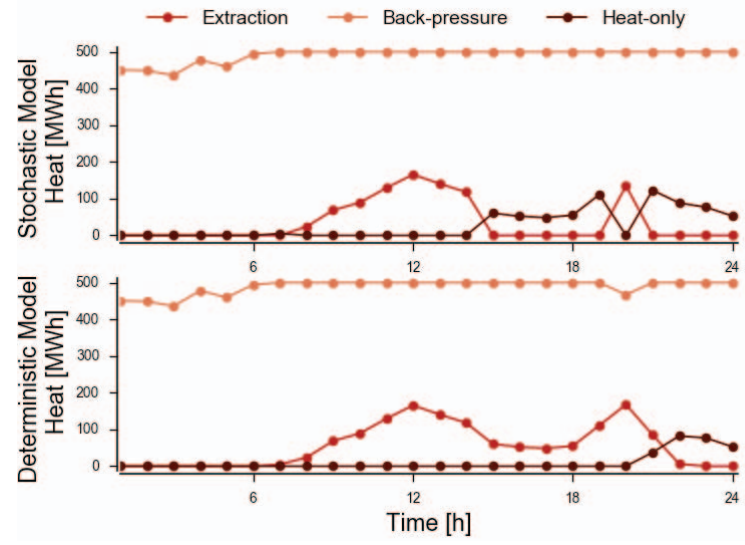

Fig. 4. Stochastic and nominal hierarchical models: Initial heat dispatch

Figure 5 presents the electricity dispatch and heat redispatch for a specific scenario. The back-pressure unit is used to cover base-load, and the heat-only unit as a peaker. During time periods 7-10 the electricity output of the extraction unit is at its maximum and the electricity/heat ratio varies in order to adjust the heat production. This shows that the heat dispatch is performed optimally, taking into account the electricity market needs, contrary to a traditional heat-demand-driven dispatch. In that sense the heat sector provides flexibility to the power system by committing the most flexible extraction units at an optimal level.

\section{CONCLusion}

The model proposed in this paper provides a method for optimal heat dispatch in the context of large penetration of wind power and CHPs. This problem is formulated as a stochastic MPEC where the heat system operator minimizes the heat production cost, subject to constraints representing various scenarios of day-ahead electricity market clearing. The 


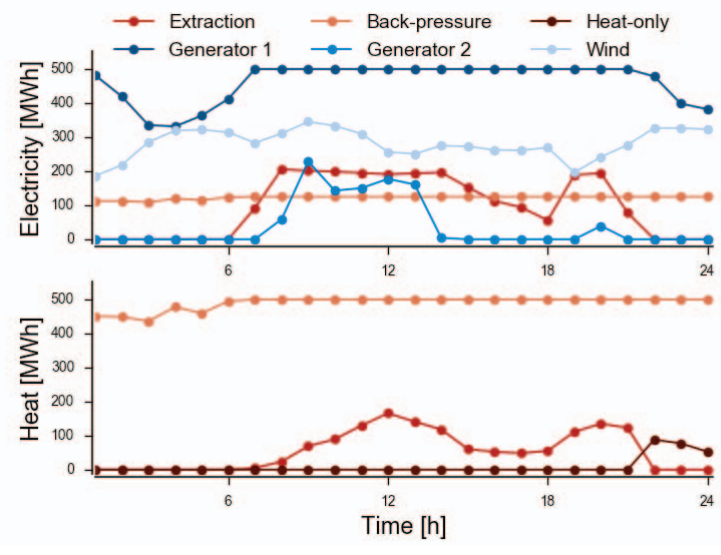

Fig. 5. Stochastic hierarchical model: Electricity dispatch and heat final dispatch for a specific scenario $\left(\nu_{1}, \alpha_{g_{1}}=37 \$ / M W h, \alpha_{g_{2}}=69 \$ / M W h\right)$

uncertainties on the electricity market are modelled through a finite set of scenarios for wind power production, electricity demand and rival participants' offers.

The hierarchical structure allows the heat system operator to model explicitly the effect of the participation of CHPs on the day-ahead electricity market. This is crucial in order to compute the actual heat merit order and make informed decisions. Additionally this coordination of heat and electricity systems allows the heat system to provide more flexibility to the power system by exploiting optimally the flexibility of extraction units. This approach can help mitigate the impact of inflexible CHP units in the power sector and reduce wind curtailment. Moreover, contrary to co-clearing of heat and electricity approaches proposed in the literature, this method provides a decision-making tool for heat system operators applicable to the current sequential market framework.

This work opens up several directions for future research. First of all it is important to assess the benefits of this method in terms of increased flexibility and wind power integration. For that purpose we should compare it to other approaches, such as co-clearing, and existing decoupled deterministic market clearing. We should also asses out-of-sample the benefits of including uncertainty in the decision-making. Additionally the proposed model can be upgraded by introducing heat storage and heat pumps in order to allow inter-temporal and fuel arbitrages; thus providing more flexibility to the overall system. Including network constraints can be another natural extension. Furthermore MPECs are complex non-linear models that can become intractable as the size of the system and the number of scenarios increase. In order to implement this model on large-scale heat and power systems and integrate a larger number of scenarios that capture accurately the uncertainties we can use decomposition techniques as suggested in [18]. Finally including new products in the day-ahead electricity market clearing, such as block and flexible orders, as well as modelling the balancing stage can be interesting extensions to this model.

\section{ACKNOWLEDGEMENT}

This work was partly supported by the Danish Energy Development Programme (EUDP) through the EnergyLab Nordhavn project (Grant id: EUDP 64015-0055). The authors are grateful to J. Boldt from HOFOR for supporting with discussions. The authors also wish to acknowledge Dr. Jalal Kazempour (DTU), Tue Vissing Jensen (DTU) and Nicolò Mazzi (Unipd) for discussion on previous versions of this work, as well as two anonymous reviewers for their valuable feedbacks.

\section{REFERENCES}

[1] H. Lund, B. Möller, B. V. Mathiesen, and A. Dyrelund, "The role of district heating in future renewable energy systems," Energy, vol. 35, no. 3, pp. 1381-1390, 2010.

[2] P. Meibom, K. B. Hilger, H. Madsen, and D. Vinther, "Energy comes together in denmark: The key to a future fossil-free danish power system," IEEE Power Energy Mag., vol. 11, no. 5, pp. 46-55, 2013.

[3] X. Chen, C. Kang, M. O’Malley, Q. Xia, J. Bai, C. Liu, R. Sun, W. Wang, and $\mathrm{H}$. Li, "Increasing the flexibility of combined heat and power for wind power integration in china: Modeling and implications," IEEE Trans. Power Syst., vol. 30, no. 4, pp. 1848-1857, 2015.

[4] B. C. Ummels, M. Gibescu, E. Pelgrum, W. L. Kling, and A. J. Brand, "Impacts of wind power on thermal generation unit commitment and dispatch," IEEE Trans. Energy Convers., vol. 22, no. 1, pp. 44-51, 2007.

[5] Z. Li, W. Wu, M. Shahidehpour, J. Wang, and B. Zhang, "Combined heat and power dispatch considering pipeline energy storage of district heating network," IEEE Trans. Sustain. Energy, vol. 7, no. 1, pp. 12-22, 2016.

[6] B. Rolfsman, "Combined heat-and-power plants and district heating in a deregulated electricity market," Appl. Energy, vol. 78, no. 1, pp. 37-52, 2004.

[7] M. Zugno, J. M. Morales, and H. Madsen, "Commitment and dispatch of heat and power units via affinely adjustable robust optimization," arXiv preprint arXiv:1507.05811, 2015.

[8] L. Maurovich-Horvat, P. Rocha, and A. S. Siddiqui, "Optimal operation of combined heat and power under uncertainty and risk aversion," Energy Build., vol. 110, pp. 415-425, 2016.

[9] L. Baringo and A. J. Conejo, "Offering strategy of wind-power producer: A multi-stage risk-constrained approach," IEEE Trans. Power Syst., vol. 31, no. 2, pp. 1420-1429, 2015.

[10] M. Zugno, J. M. Morales, P. Pinson, and H. Madsen, "Pool strategy of a price-maker wind power producer," IEEE Trans. Power Syst., vol. 28, no. 3, pp. 3440-3450, 2013.

[11] R. Lahdelma and H. Hakonen, "An efficient linear programming algorithm for combined heat and power production," Eur. J. Oper. Res., vol. 148, no. 1, pp. 141-151, 2003.

[12] S. A. Gabriel, A. J. Conejo, J. D. Fuller, B. F. Hobbs, and C. Ruiz, Complementarity Modeling in Energy Markets. Springer, 2012, vol. 180.

[13] J. Fortuny-Amat and B. McCarl, "A representation and economic interpretation of a two-level programming problem," J. Oper. Res. Soc., vol. 32, no. 9, pp. 783-792, 1981.

[14] S. Siddiqui and S. A. Gabriel, "An SOS1-based approach for solving MPECs with a natural gas market application," Netw. Spat. Econ., vol. 13, no. 2, pp. 205-227, 2013.

[15] W. Bukhsh, "Data for stochastic multiperiod optimal power flow problem.” [Online]. Available: http://sites.google.com/site/datasmopf/

[16] H. Madsen, "Time series analysis. course notes." [Online]. Available: http://www.imm.dtu.dk/ hmad/time.series.analysis/assignments/ index.html

[17] L. Mitridati, "Hierarchical heat dispatch." [Online]. Available: http: //dx.doi.org/10.5281/zenodo.55580

[18] L. Baringo and A. J. Conejo, "Wind power investment: A benders decomposition approach," IEEE Trans. Power Syst., vol. 27, no. 1, pp. 433-441, 2012. 